\title{
1 Deletion of MFGE8 inhibits neointima formation upon arterial damage
}

2

Viola: MFGE8 promotes neointima formation upon arterial damage

Joana R. Viola, $\mathrm{PhD}^{1,2,3}$, Patricia Lemnitzer, $\mathrm{BSc}^{1}$, Nicole Paulin, VetD ${ }^{1}$, Maik Drechsler, $\mathrm{PhD}^{1,2,3}$, Maliheh Nazari-Jahantigh, $\mathrm{PhD}^{1,3}$, Sanne Maas, MSc${ }^{1}$, Renske J. De Jong, $\mathrm{PhD}^{1}$, Janine Winter, BSc', Andreas Schober, MD ${ }^{1}$, Christian Weber, MD ${ }^{1,3}$, Kamran Atabai, MD ${ }^{4}$, Oliver Soehnlein, MD, PhD

${ }^{1}$ Institute for Cardiovascular Prevention (IPEK), LMU Munich, Germany

${ }^{2}$ Academic Medical Center (AMC), Department of Pathology, Amsterdam University, the Netherlands

${ }^{3}$ DZHK, partner site Munich Heart Alliance, Germany

${ }^{4}$ Department of Medicine, Cardiovascular Research Institute, Lung Biology Center, University of California, San Francisco, California, USA.

${ }^{5}$ Department of Physiology and Pharmacology (FyFa), Karolinska Institutet, Stockholm, Sweden.

Correspondence:

Oliver Soehnlein

IPEK, LMU Munich

Pettenkoferstr. 9

80336 Munich

Phone +49-(0)89-4400-54677

Fax +49-(0)89-4400-54352

Email: oliver.soehnlein@gmail.com

Word count: 699 
Dear Sirs,

Atherosclerosis, a chronic inflammation of the vessel wall, is a major cause for vascular mortality due to narrowing (stenosis) of the arterial wall. In part, artery stenosis is therapeutically addressed by widening the vessel through angioplasty. Although well-established, angioplasty can damage the arterial endothelium, giving rise to an inflammatory response that leads to a neointimal hyperplasia with consequent recurrence of stenosis $(1,2)$. Key players in this process are leukocytes and smooth muscle cells (SMCs), as leukocyte recruitment and smooth muscle cell proliferation and migration are determinants of neointimal hyperplasia (3).

MFGE8 (Milk Fat Globule-Epidermal Growth factor 8) or lactadherin is mostly regarded as a bridging molecule with a critical function in efferocytosis and, hence, during resolution of inflammation (4). However, MFGE8 also plays a major role in promoting neovascularization (5), and, more recently, arterial MFGE8 expression emerged as a molecular hallmark of adverse cardiovascular remodeling upon ageing (6). In this context, MFGE8 is directly associated with SMC proliferation and migration $(6,7)$, suggesting its participation in neointima formation. We here show that MFGE8 negatively impacts on arterial restenosis and its neutralization may therefore be a potential therapeutic strategy.

To study the role of MFGE8 in arterial restenosis we subjected two groups of mice, Apoe $e^{-\gamma}$ and Apoe $e^{-1-}$ $M f g e 8^{-/}$, to wire injury of the left carotid artery. To simulate hypercholesterolemia, a condition often present in atherosclerotic patients, the mice were fed a high fat diet (HFD), starting one week prior to injury. Two weeks after the injury the mice were euthanized and the blood and carotids were collected. To assess neointima formation, the carotids, were stained for elastic tissue fibers with Verhoeff's van Gieson stain (EVG). Neointima area was significantly smaller throughout the injured carotid in mice lacking MFGE8 as compared to the Apoe $e^{-/}$group (Figure 1, A-C). No differences in blood counts were observed between the groups (Table 1, Supplemental Data), suggesting that the distinct neointima areas are mediated by local cells. However, cholesterol levels in the blood were higher in Apoe $\mathrm{efged}^{-/ /}$mice as compared to Apoe $e^{-/}$; likely a consequence of decreased fatty acid uptake by the liver as well as small intestine, as MFGE8 has been reported to promote fatty acid uptake $(8,9)$. To determine which cells contributed the most for the larger neointima observed in Apoe ${ }^{-/}$mice, we stained the carotids with antibodies against macrophages and SMCs, since MFGE8 has been reported to be expressed in these cells $(10,11)$. Lack of MFGE8 did not affect neointimal macrophages, while SMC areas were vastly reduced (Figure 1D, E). To assess whether this was the result of reduced cell proliferation, the carotid arteries were stained with an antibody against Ki67, a cellular marker of proliferation. The staining revealed significantly less SMC proliferation in mice lacking MFGE8 as compared to their wild type littermates, suggesting a determinant role of lactadherin in restenosis formation. To confirm this observation, and verify MFGE8 as a therapeutic target, we subjected Apoe $e^{-/-}$mice to arterial injury combined with the local application of siRNA, either against MFGE8 or scrambled. siRNA against MFGE8 resulted in 50\% reduced expression of the protein both in the intima and the neointima (lesion) area of the carotid aretery (Figure 1, Supplemental Data). Similar to what was observed in the knockout animal models, analysis of the injured carotids of mice treated with siRNA directed to MFGE8 showed a decreased neointima sizes as compared to mice treated with control siRNA (Figure 1, G-I). Equally in accordance to the studies in the knockout animal models, no differences in blood counts were observed between the groups (Table 1, Supplemental Data). Blood cholesterol levels remained unchanged (Figure 2, Supplemental Data) supporting the argument supra presented for the difference observed in the blood of the knockout animal models, since the siRNA effect is strictly local it does not affect fatty acid uptake. Consistent with the observations in the carotids of Apoe $^{-/}$vs. Apoe $\mathrm{Mfge}^{-/}$mice, the administration of siRNA against MFGE8 affected SMCs content in the neointima (Figure 1K) and its proliferation (Figure 1L) but the macrophage composition remained unchanged (Figure 1J).

Overall these results strongly point towards a relevant role of MFGE8 in post-injury arterial wall remodeling, with the potential to be exploited for therapeutic purposes. Our studies suggest these effects to be SMC-mediated, more specifically: by stimulating SMC proliferation MFGE8 promotes the formation of neointima possibly leading to hyperplasia and consequent stenosis.

80 
81 Conflicts of interest

82 None declared.

83

84

85 This study was supported by the German Research Foundation (SO876/6-1, SO876/11-1, SFB914 B08,

86 SFB1123 A06 \& B05), the Vetenskapsrådet (2017-01762), the NWO (VIDI project 91712303), the

87 European Union's Horizon 2020 research and innovation programme under the Marie Skłodowska-Curie

88 grant agreement No 675111, and the FöFoLe program of the medical faculty of the LMU Munich. 
90 1. Dangas G, Kuepper F. Cardiology patient page. Restenosis: repeat narrowing of a coronary artery: prevention and treatment. Circulation 2002; 105(22): 2586-7.

92 2. Nicolais C, Lakhter V, Virk HUH, et al. Therapeutic Options for In-Stent Restenosis. Curr Cardiol 93 Rep 2018; 20(2): 7.

94 3. Soehnlein O, Wantha S, Simsekyilmaz S, et al. Neutrophil-derived cathelicidin protects from 95 neointimal hyperplasia. Sci Transl Med 2011; 3(103): 103 ra98.

96 4. Hanayama R, Tanaka M, Miwa K, et al. Identification of a factor that links apoptotic cells to 97 phagocytes. Nature 2002; 417(6885): 182-7.

98 5. Silvestre JS, Thery C, Hamard G, et al. Lactadherin promotes VEGF-dependent 99 neovascularization. Nat Med 2005; 11(5): 499-506.

100 6. Fu Z, Wang M, Gucek M, et al. Milk fat globule protein epidermal growth factor-8: a pivotal relay 101 element within the angiotensin II and monocyte chemoattractant protein-1 signaling cascade mediating 102 vascular smooth muscle cells invasion. Circ Res 2009; 104(12): 1337-46.

103 7. Wang M, Fu Z, Wu J, et al. MFG-E8 activates proliferation of vascular smooth muscle cells via 104 integrin signaling. Aging Cell 2012; 11(3): 500-8.

1058 8halifeh-Soltani A, Gupta D, Ha A, et al. Mfge8 regulates enterocyte lipid storage by promoting 106 enterocyte triglyceride hydrolase activity. JCI Insight 2016; 1(18): e87418.

107 9. Khalifeh-Soltani A, McKleroy W, Sakuma S, et al. Mfge8 promotes obesity by mediating the 108 uptake of dietary fats and serum fatty acids. Nat Med 2014; 20(2): 175-83.

109 10. Bagnato C, Thumar J, Mayya V, et al. Proteomics analysis of human coronary atherosclerotic 110 plaque: a feasibility study of direct tissue proteomics by liquid chromatography and tandem mass 111 spectrometry. Mol Cell Proteomics 2007; 6(6): 1088-102.

112 11. Ait-Oufella H, Kinugawa K, Zoll J, et al. Lactadherin deficiency leads to apoptotic cell 113 accumulation and accelerated atherosclerosis in mice. Circulation 2007; 115(16): 2168-77. 
A

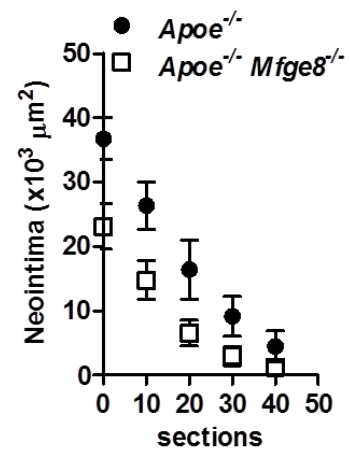

D

G
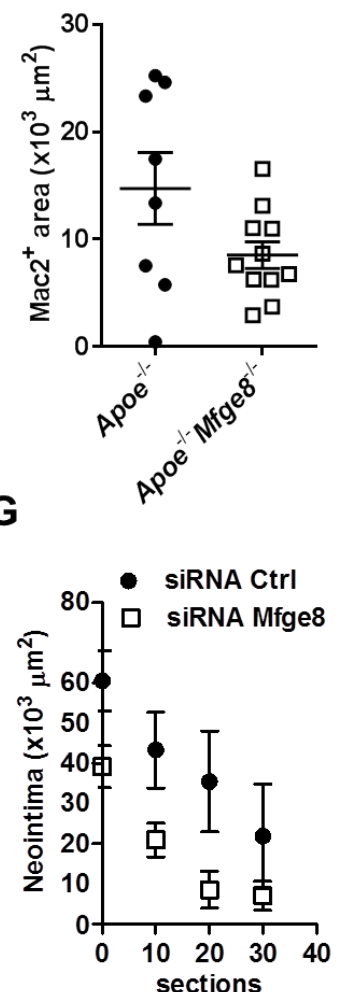

J

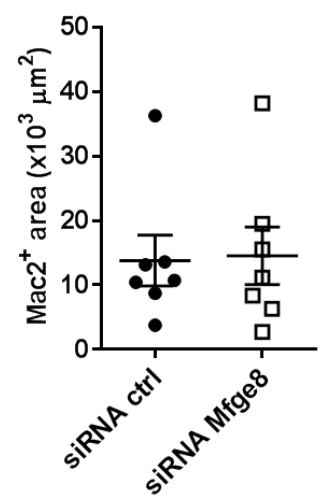

B

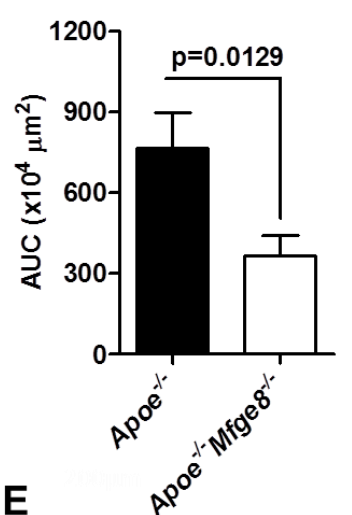

E
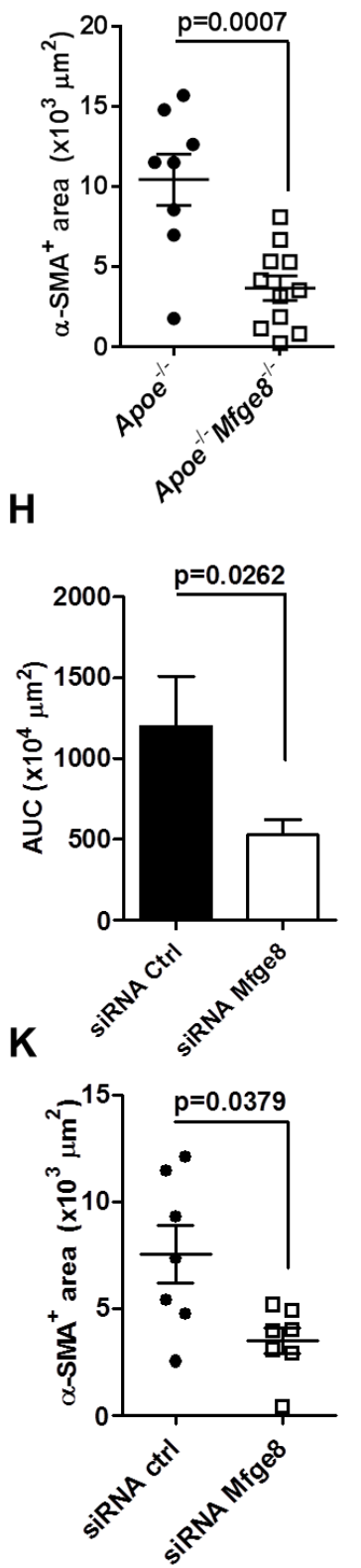

C

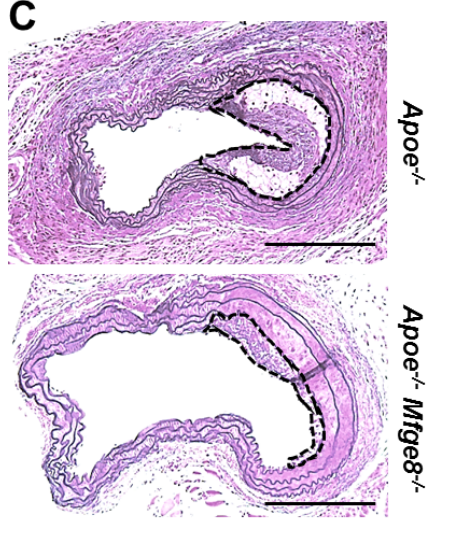

F

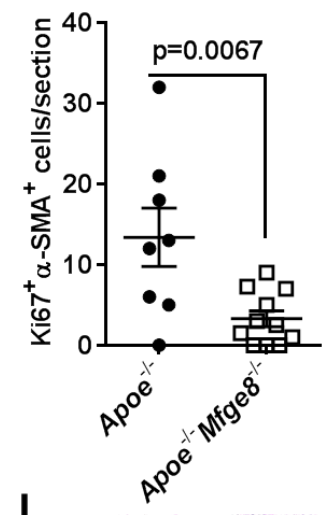

I
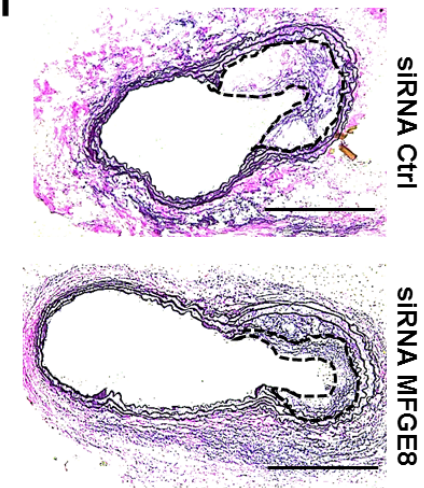

L

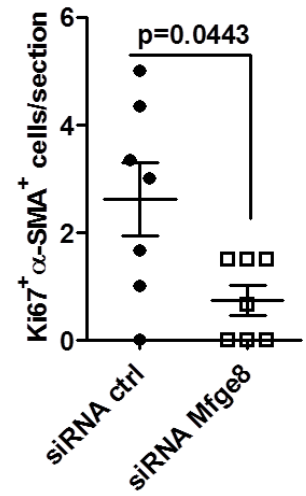


118 Figure 1. Mfge8 promotes post-injury neointima formation by fostering smooth muscle cell 119 proliferation. Mice were fed a high fat diet (HFD) starting one week prior to carotid injury induction. 120 Two weeks after injury, mice were euthanized and the blood and carotids were collected. Carotids were 121 fixed in paraformaldehyde (PFA) and embedded in paraffin. For each antibody staining three sections per 122 mouse were analyzed. (A-F) Assessment of neointima size and composition in Apoe versus Apoe $^{-/-}$ $123 \mathrm{Mfge}^{-/}$mice. (A) Neointima area was analyzed by elastic tissue fibers staining, with Verhoeff's van 124 Gieson stain (EVG), and quantified throughout the carotids. (B) To better quantify the difference in 125 neointima area between the two groups, the area under the curve (AUC), corresponding to the data points presented in (A), was calculated. (C) Representative images of EVG stained injured carotids. To identify possible differences in neointima composition between the two mice groups, carotids were stained with antibodies against (D) macrophages (anti-Galactin-3 or anti-Mac2) or (E) smooth muscle cells (SMCs) (anti- $\alpha$-SMA). Each data point indicates the average of positive area per section in one mouse. To determine whether the increased numbers of SMCs in the neointima of Apoe $\mathrm{Mfge}^{-/}$mice originated from cell proliferation or migration, carotids were stained with an antibody against a cell proliferation marker, Ki67 (F). $\mathrm{n}=8$ (Apoe (- $^{-\alpha}$ mice) or $\mathrm{n}=11$ (Apoe Afge $^{-/-}$mice) throughout panels A-F. Statistical comparisons were made with t-test following D'Agostino Pearson omnibus normality test. (G-L) Assessment of neointima size and composition in Apoe $e^{-/-}$mice treated with control siRNA or directed to Mfge8. (G) Neointima area was quantified throughout the carotids, and $(\mathrm{H})$ the AUC, corresponding to the data points presented in $(\mathrm{G})$, was calculated. (I) Representative images of EVG stained injured carotids. Carotids were stained with antibodies against (D) macrophages (anti-Galactin-3 or anti-Mac2) or (E) SMCs (anti- $\alpha$-SMA). Each data point indicates the average of positive area per section in one mouse. (F) Carotids were stained with an antibody against a cell proliferation marker, Ki67. $n=7$ mice per group throughout panels G-L. Statistical comparisons were made with t-test following Kolmogorov-Smirnov 142 (KS) normality test. All data is represented as mean \pm SEM. Scale bar represents $200 \mu \mathrm{m}$. 
165 Fax +49-(0)89-4400-54352

166 Email: oliver.soehnlein@gmail.com

${ }^{1}$ Institute for Cardiovascular Prevention (IPEK), LMU Munich, Germany

${ }^{2}$ Academic Medical Center (AMC), Department of Pathology, Amsterdam University, the Netherlands

${ }^{3}$ DZHK, partner site Munich Heart Alliance, Germany

${ }^{4}$ Department of Medicine, Cardiovascular Research Institute, Lung Biology Center, University of California, San Francisco, California, USA.

${ }^{5}$ Department of Physiology and Pharmacology (FyFa), Karolinska Institutet, Stockholm, Sweden.

170 
A

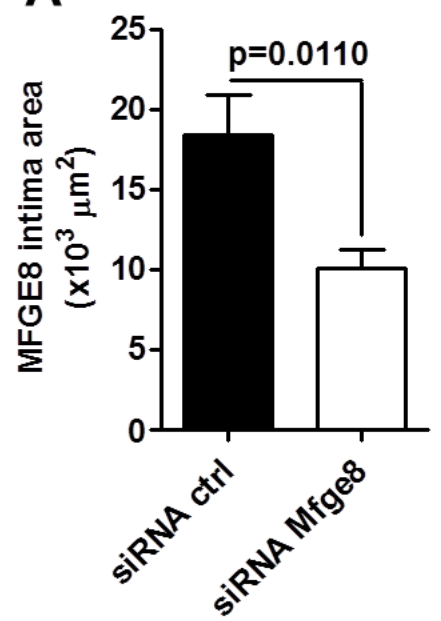

B

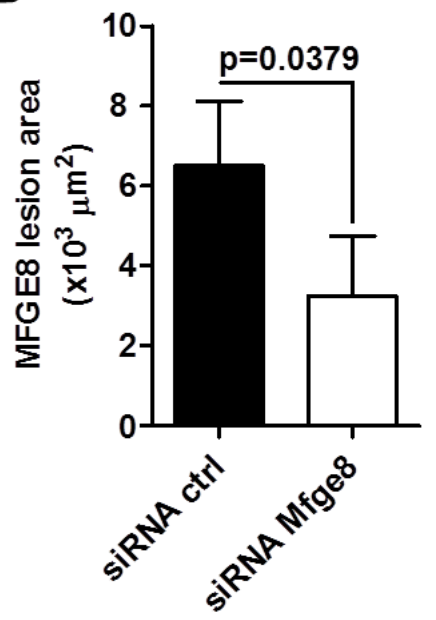

171

\section{Supplementary Figure 1. Delivery of siRNA against MFGE8 decreases MFGE8 expression in the} carotids by 50\%. Mice were fed a high fat diet (HFD) starting one week prior to carotid injury induction. siRNA (Accell siRNA, $4 \mathrm{nmol} /$ treatment, Dharmacon), formulated with pluronic gel (35\%) as previously described (1), was locally administered one week after injury. Two weeks after injury, mice were euthanized and the blood and carotids were collected. Carotids were fixed in paraformaldehyde (PFA) and embedded in paraffin. Three sections, per mouse, along the carotids were stained with antibody against MFGE8. The protein expression was quantified in (A) the intima and (B) in the lesion (neointima area) and is presented as positive area per section per mouse. $n=7$. Statistical comparisons were made with $t$-test following Kolmogorov-Smirnov (KS) normality test. All data is represented as mean \pm SEM. 

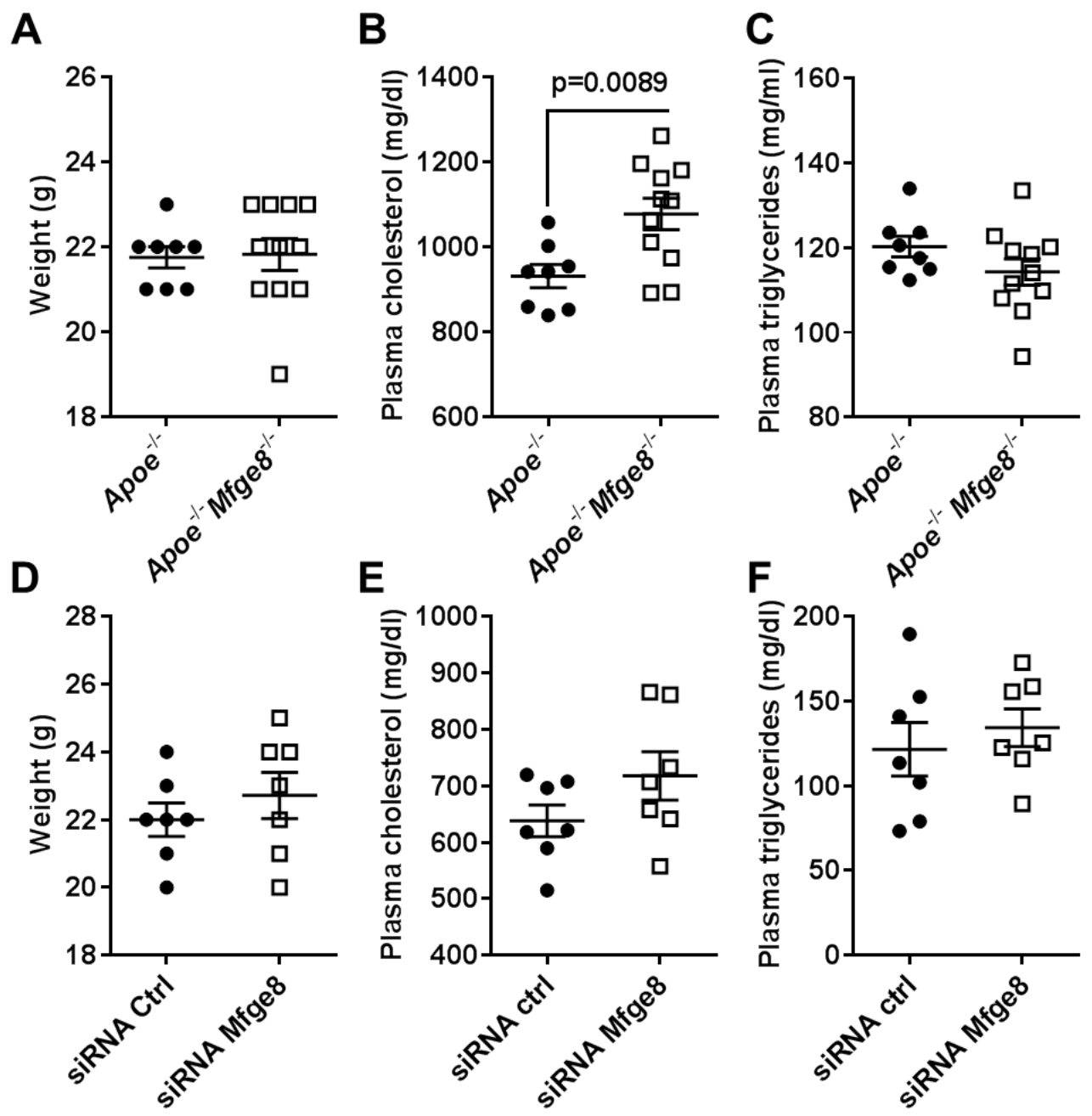

182
Supplementary Figure 2. Lack of MFGE8 does not affect body weight and plasma lipid levels. Mice were fed a high fat diet (HFD) starting one week prior to carotid injury induction. Two weeks after injury, mice were euthanized and the blood and carotids were collected. (A-C) Initial studies were conducted with knockout murine models: Apoe $e^{-/-}$mice versus Apoe $\mathrm{Mfge}^{-/-}$mice. (A) Prior to euthanasia, mice were weighted. Plasma levels of (B) cholesterol and (C) triglycerides were quantified. $\mathrm{n}=8$ (Apoe $e^{-/-}$mice) or $\mathrm{n}=11$ (Apoe ${ }^{-/} \mathrm{Mfge}^{-/-}$mice). Statistical comparisons were made with t-test following D'Agostino Pearson omnibus normality test. (D-F) To confirm the initial findings, and verify MFGE8 as a therapeutic target, we subjected Apoe ${ }^{-/-}$mice to carotid injury in combination with local administration of siRNA, either control or directed to MFGE8. (D) Prior to euthanasia mice were weighted. Thereafter, plasma levels of (B) cholesterol and (C) triglycerides were quantified. $\mathrm{n}=7$. Statistical comparisons were made with t-test following Kolmogorov-Smirnov (KS) normality test. All data is represented as mean \pm SEM. 


\section{References}

Supplementary Table 1. Lack of MFGE8 does not affect white blood cell counts. Mice were subjected to injury in the left carotid and euthanized two weeks later. Blood was collected and blood cell counts was evaluated by flow cytometry. Cells were stained with a combination of antibodies (CD45, CD115, CD11b, $\mathrm{CD} 3, \mathrm{CD} 4, \mathrm{CD} 8, \mathrm{Gr} 1)$. Results are presented as number of cells/ml.

210 1. Nazari-Jahantigh M, Wei Y, Noels H, et al. MicroRNA-155 promotes atherosclerosis by repressing Bc16 in macrophages. J Clin Invest 2012; 122(11): 4190-202. 